\title{
Community-Acquired Pneumonia in Older Veterans: Does the Pneumonia Prognosis Index Help?
}

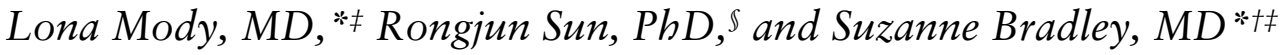

OBJECTIVES: Mortality rates from pneumonia increase steadily with age. Recently, a disease severity model (the Pneumonia Prognosis Index (PPI)) has been developed to predict mortality from community-acquired pneumonia (CAP). PPI ranks severity of pneumonia from 1 to 5 , with 5 being most severe. This retrospective study utilizes the PPI to address the prognosis of CAP in older adults.

DESIGN: Retrospective review of medical charts.

SETTING: Department of Veterans Affairs Medical Center. PARTICIPANTS: All adults aged 60 and older admitted to a Veterans Affairs Medical Center with CAP between January 1 and December 31, 1998.

MEASUREMENTS: PPI was calculated using subjects' demographics, comorbidities, presenting symptoms, and laboratory measurements.

RESULTS: Eighty-two patients aged 60 and older were admitted with 101 episodes of CAP. The mean age \pm standard deviation was $72 \pm 9$. Seventy-four episodes were admitted from the emergency room, 20 from another hospital, seven from nursing homes, and five from outpatient clinics. Mean length of stay was $7.1 \pm 6$ days. Comorbid conditions included coronary artery disease, diabetes mellitus, congestive heart failure, neoplasm, stroke, and renal failure. Most episodes fell into higher PPI classes, with $20 \%$ in Class 3, 46\% in Class 4, and 32\% in Class 5. PPI score was significantly related to length of stay $(P<.001)$, intensity of care $(P<.0001)$, and presence of complications $(P<$ $.001)$. Mortality was $14 \%$ at 30 days: $0.5 \%$ in Class 3, $10.8 \%$ in Class 4 , and $25 \%$ in Class 5 .

CONCLUSION: The PPI was effective in identifying older adults with CAP who were at risk of a poor outcome, but

From the "Divisions of Geriatric Medicine and Infectious Diseases, the University of Michigan Medical School, Ann Arbor, Michigan; ${ }^{\ddagger}$ GRECC, Veterans Affairs Ann Arbor Healthcare System, Ann Arbor, Michigan; and Institute of Gerontology, University of Michigan, Ann Arbor, Michigan.

Preliminary results of this paper were presented at the Annual Meeting of American Geriatrics Society, 2000, Nashville, Tennessee, and the Immunocompromised Host Society Meeting, 2000, Halifax, Nova Scotia.

Address correspondence to Dr. Lona Mody, GRECC 11 G, Veterans Affairs Healthcare System, 2215 Fuller Road, Ann Arbor, MI 48105. E-mail: lonamody@umich.edu the practical utility of this index remains to be determined. Further prospective studies are required to elucidate the importance of comorbidities, severity at presentation, and premorbid functional status on clinical and functional outcomes of CAP in older adults. J Am Geriatr Soc 50:434438, 2002.

Key words: community acquired pneumonia; pneumonia prognosis index; older adults

$\mathrm{P}$ neumonia and influenza combined are the fifth leading cause of death in the United States. ${ }^{1}$ Four million cases of pneumonia occur annually, and $20 \%$ of episodes require hospitalization. ${ }^{2}$ The average length of stay for pneumonia in individuals aged 65 and older is 9.4 days, compared with 6.2 days for those younger than $65 .^{3}$ Mortality rates from pneumonia increase steadily with age, from a rate of 24 per 100,000 admissions for patients aged 60 to 64 to 1,032 per 100,000 admissions for those aged 85 and older. ${ }^{4}$

Hazards of hospitalization have been well documented in older adults. ${ }^{5}$ Functional decline occurs quickly upon hospitalization and often fails to improve after discharge. ${ }^{6,7}$

Recently several studies have been performed to define pathways of care for individuals with community-acquired pneumonia (CAP). Fine et al. developed a disease-severity model, the Pneumonia Prognosis Index (PPI), based on mortality. ${ }^{8}$ However, whether this PPI and the proposed recommendations can be applied to older adults with CAP has not been specifically addressed. Age carries a significant weight in assessing the severity of illness by Fine's criteria. We applied Fine's PPI retrospectively to all veterans who were admitted with CAP over a 1-year period to assess the validity of the PPI for older adults.

\section{METHODS}

\section{Study Population}

We studied all cases of CAP in patients aged 60 and older admitted to the Ann Arbor Veterans Affairs Medical Center between January 1, 1998, and December 31, 1998. Patients were identified by admitting and discharge diagnoses from computerized records. 
To be considered, individuals had to be aged 60 and older and have pneumonia defined by chest radiograph demonstrating a new infiltrate and the presence of at least two of the following symptoms and signs compatible with pneumonia: (1) new or increased cough, (2) new or increased sputum production, (3) fever of $38^{\circ} \mathrm{C}$ or greater, (4) pleuritic chest pain, (5) new or increased physical findings on chest examination (rales, rhonchi, wheezes, bronchial breath sounds), (6) new/increased shortness of breath, (7) respiratory rate of 25 or more per minute, or (8) worsening mental or functional status. ${ }^{9}$ Episodes that did not meet the case definition of pneumonia were excluded even if it was recorded as the principal diagnosis. We also excluded all individuals who acquired pneumonia more than 72 hours after admission to hospital.

\section{Demographic Data and Clinical Parameters}

Demographic data of age, gender, site of residence, admitting diagnosis, length of stay, medical history (abnormal mental status, fever, cough, pleuritic chest pain, falls, hemoptysis, antibiotic usage, reduced oral intake), signs on admission (temperature, heart rate, respiratory rate, blood pressure), laboratory parameters within 24 hours of admission (serum urea nitrogen, sodium, glucose, white cell count, hematocrit, partial pressure of arterial oxygen $\left(\mathrm{P}_{\mathrm{a}} \mathrm{O}_{2}\right)$, and arterial $\mathrm{pH}$ ), radiographic findings, complications, and outcome were recorded. The presence of preexisting comorbidities, including neoplasm, congestive heart failure (CHF), cerebrovascular disease, renal disease, liver disease, diabetes mellitus, coronary artery disease, and chronic obstructive pulmonary disease (COPD), were assessed by past medical history, medication history, and review of laboratory data. Neoplastic disease included any cancer except skin cancer that was active at the time of presentation or diagnosed within a year of presentation.

\section{Microbiology}

Microbiology data was obtained from clinical records. A significant proportion of patients (20 episodes) were admitted from outside hospitals; microbiology data on these individuals were not available.

\section{Pneumonia Prognosis Index}

The PPI by Fine et al. was utilized, and patients were classified as being in one of the five classes (see Table 1). Class 1 (aged $<50$, no coexisting conditions, laboratory abnormalities, or physical findings), Class 2 ( $<70$ points), Class 3 (71-90 points), Class 4 (90-130 points) and Class 5 (>130 points). ${ }^{8}$

Table 1. Pneumonia Prognosis Index ${ }^{8}$

\begin{tabular}{|c|c|}
\hline Characteristic & Points Assigned \\
\hline \multicolumn{2}{|l|}{ Age } \\
\hline Men & Age, years \\
\hline Women & Age -10 , years \\
\hline Nursing home resident & +10 \\
\hline \multicolumn{2}{|l|}{ Coexisting illness } \\
\hline Neoplastic disease & +30 \\
\hline Liver disease & +20 \\
\hline Congestive heart failure & +10 \\
\hline Cerebrovascular disease & +10 \\
\hline Renal disease & +10 \\
\hline \multicolumn{2}{|l|}{ Physical examination findings } \\
\hline Altered mental status & +20 \\
\hline Respiratory rate $\geq 30 / \mathrm{min}$ & +20 \\
\hline Systolic blood pressure $<90 \mathrm{mmHg}$ & +20 \\
\hline Temperature $<35^{\circ} \mathrm{C}$ or $\geq 40^{\circ} \mathrm{C}$ & +15 \\
\hline Pulse $\geq 125 / \mathrm{min}$ & +10 \\
\hline \multicolumn{2}{|l|}{ Laboratory and radiographic findings } \\
\hline Arterial $\mathrm{pH}<7.35$ & +30 \\
\hline Blood urea nitrogen $\geq 30 \mathrm{mg} / \mathrm{dL}$ & +20 \\
\hline Sodium $<130 \mathrm{mEq} / \mathrm{dL}$ & +20 \\
\hline Glucose $\geq 250 \mathrm{mg} / \mathrm{dL}$ & +10 \\
\hline Hematocrit $<30 \%$ & +10 \\
\hline Partial pressure of arterial oxygen $<60 \mathrm{mmHg}$ & +10 \\
\hline Pleural effusion & +10 \\
\hline Total points & - \\
\hline \multicolumn{2}{|l|}{ Class assignment } \\
\hline Class 1 & Outpatient $\mathrm{Rx}$ with absent physical findings \\
\hline Class 2 & $\leq 70$ \\
\hline Class 3 & $71-90$ \\
\hline Class 4 & $91-130$ \\
\hline Class 5 & $>130$ \\
\hline
\end{tabular}




\section{Outcomes}

The primary outcome was mortality at 30 days after the diagnosis of pneumonia. Secondary outcomes were length of stay, admission to a monitored bed, and the presence of complications from pneumonia defined by empyema, exacerbation of CHF, myocardial infarction, shock, acute respiratory distress syndrome, intubation, and metastatic infection.

\section{Data Analysis}

Data were entered in Epi-Info 6 (Version 6.04b, Centers for Disease Control, Atlanta, GA) and analyzed in SAS, version 6.12 (SAS Institute, Inc., Cary, NC). All the residents were stratified into Class 1 to 5 based on their PPI score. The unit of analysis was an episode of pneumonia, because some of the residents had more than one episode. Data were expressed as mean \pm standard deviation. Mean values were compared using analysis of variance. Categorical variables were compared using chi-square analysis. Kaplan-Meier analysis method was used to plot survival. Of the 93 discharged patients, only three were in Class 2, and 19 were in Class 3, of whom only one died in the follow-up period. Therefore, we focused our analysis on the patients in Classes 4 and 5.

\section{RESULTS}

\section{Demographics}

Between January 1, 1998, and December 31, 1998, 82 patients aged 60 and older with 101 episodes of CAP were admitted to the Ann Arbor VA Medical Center. The mean age was $72 \pm 9$ (range 60-89), and 98\% (n=98) were men. Although most of these patients were admitted through the emergency room, $20 \%(n=20)$ were transfers from an outside hospital and $7 \%(\mathrm{n}=7)$ were admitted from a nursing home. For $82 \%$ of the episodes, patients were admitted to general medical wards. The mean length of stay was 7.1 \pm 6.0 days. The mean length intensive care unit (ICU) stay for those admitted to an ICU was $4.5 \pm 2.8$ days. Only one patient was admitted for an extended period of 181 days and was excluded from the analysis of length of stay. Comorbidities were frequently present and included COPD, coronary artery disease, prior history of pneumonia, diabetes mellitus, smoking, and CHF (Table 2).

Table 2. Prevalence of Comorbidities in Older Adults Hospitalized with Community-Acquired Pneumonia

\begin{tabular}{lc}
\hline \multicolumn{1}{c}{ Comorbidities } & Episodes N = 101 \\
\hline Chronic obstructive pulmonary disease & 67 \\
Coronary artery disease & 49 \\
Current smoker & 32 \\
Congestive heart failure & 27 \\
Neoplasm & 26 \\
Cerebrovascular disease & 16 \\
Renal insufficiency & 12 \\
\hline
\end{tabular}

\section{Clinical Presentation}

The most common presenting symptoms were reduced ambulation $(45 \%)$, poor oral intake $(40 \%)$, altered mental status $(28 \%)$, pleurisy $(20 \%)$, and tachypnea $(19 \%)$. The most common presenting laboratory abnormalities were hypoxia with a $\mathrm{P}_{\mathrm{a}} \mathrm{O}_{2}$ less than $60 \mathrm{mmHg}(65 \%)$, a blood urea nitrogen greater than $30 \mathrm{mg} / \mathrm{dl}(25 \%)$, and a hematocrit less than $30 \%(18 \%)$. Mean white blood cell count was $13.9 \pm 6.5$ cells $/ \mathrm{mm}^{3}$.

\section{Microbiology}

Sputum cultures were performed in 62 episodes and showed a predominant organism in 21 . The organisms isolated were Hemophilus influenzae $(\mathrm{n}=6)$, Klebsiella pneumoniae $(\mathrm{n}=$ 4), Pseudomonas aeruginosa $(\mathrm{n}=3)$, Enterobacter aerogenes $(\mathrm{n}=3)$, Streptococcus pneumoniae $(\mathrm{n}=2)$, Staphylococcus aureus $(\mathrm{n}=2)$, Serratia marcescans $(\mathrm{n}=1)$, and Escherichia coli $(\mathrm{n}=1)$. Blood cultures were performed in 82 episodes but were positive in only five $(6 \%)$, yielding $S$. aureus in two and $S$. intermedius, $K$. pneumoniae, and $P$. aeruginosa in one each.

\section{PPI Index and Clinical Outcomes}

Most episodes of CAP requiring admission to hospital fell in Class 3 (20/101), Class 4 (46/101), and Class 5 (32/ 101). There were no episodes in Class 1 and only three in Class 2. Of Class 3 episodes, there were no abnormalities in PPI-specific findings on physical examination; only $50 \%$ $(10 / 20)$ had any comorbidities and 55\% (11/20) had a PPIspecific abnormality in their laboratory or radiographic finding. Overall, a majority of episodes $(75 \%)$ in Class 3 had no or only one PPI-specific abnormality; most were classified in Class 3 based on their age alone. Nineteen of $46(42 \%)$ episodes in Class 4 did not have any comorbidities, but 31 of $32(96 \%)$ episodes in Class 5 had one or more comorbidities.

A higher PPI score was associated with a higher rate of admission to a monitored bed, either telemetry or ICU $(P<.0001)$. With a higher PPI score there was also an increased chance of developing one or more complications of pneumonia $(P<.001)$, the most prevalent being exacerbation of $\mathrm{CHF}(14 \%)$ and respiratory failure requiring intubation $(13 \%)$. Moreover, a higher PPI class predicted a longer length of stay $(P<.001)$. The overall in-hospital mortality was $8 \%(\mathrm{n}=8 / 101)$. For Classes 3,4 , and 5, the 30 -day mortalities were $0.5 \%, 10.8 \%$, and $25.0 \%$, respectively. Mean long-term follow-up of 13 months revealed mortalities of $1 \%, 33 \%$, and $63 \%$ for PPI Classes 3, 4, and 5 , respectively (Figure 1, Table 3). Thus, higher PPI score was significantly associated with higher likelihood of death in hospital $(8 \%)$, at 30 days $(14 \%)$, and at mean long-term follow-up of 13 months $(37 \%)(P<.001)$ (Table 3$)$.

\section{DISCUSSION}

The PPI has been developed in an effort to address the variability in the rates of hospitalization of adults with pneumonia. ${ }^{8}$ It is aimed at identifying patients with CAP who are at a low risk of mortality and other adverse outcomes so as to help physicians make more rational decisions about hospitalization for patients with pneumonia. In the study by Fine et al., the overall in-hospital mortality 


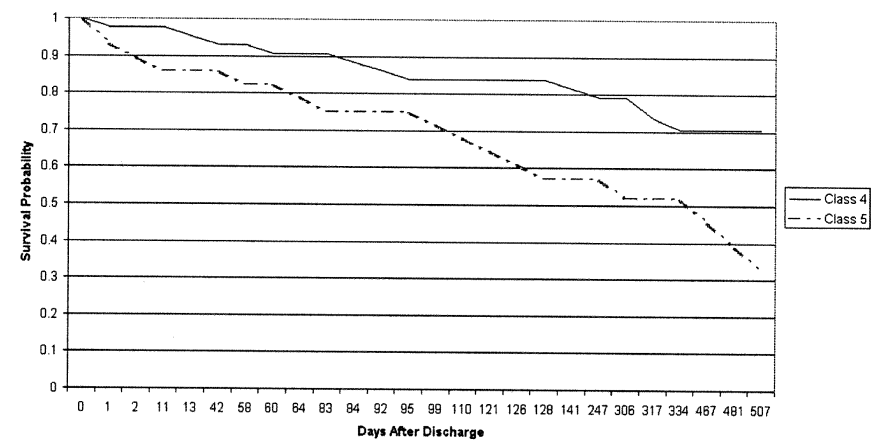

Figure 1. Kaplan-Meier plots of Class 4 and Class 5 patients.

for all adults aged 18 and older in Classes 3, 4, and 5 was $0.9 \%, 9.3 \%$, and $27 \%$, respectively. Outpatient management was recommended for PPI Classes 1 and 2, a brief observation for Class 3, and traditional in-patient care for Classes 4 and $5 .{ }^{8}$ In our study, we found that most pneumonia episodes fell into PPI Classes 3, 4, and 5.

Mortality from CAP increases steadily with age., $4,8,10-16$ In a retrospective medical record review of Medicare patients, in-hospital mortality for PPI Classes 3, 4, and 5 was $2.8 \%, 8.5 \%$, and $24.4 \%$, respectively. ${ }^{11}$ Twenty-three percent of this cohort was admitted from a nursing facility. In another study assessing the utility of this index for patients with nursing home acquired pneumonia, a higher PPI class predicted a poor outcome; 30-day mortality for Classes 3, 4 , and 5 in this cohort was $4.8 \%, 12.0 \%$, and $33.0 \%$, respectively. ${ }^{10}$ In our study, a higher PPI score was associated with an increased frequency of admission to a monitored bed, a higher incidence of developing one or more complications, a longer length of stay, and a higher risk of mortality. However, the in-hospital mortality was lower than these previous studies in older adults, with rates of $0.5 \%, 6.5 \%$, and $12.5 \%$ for Classes 3,4 , and 5 , respectively. Perhaps the better outcome can be attributed to the fact that our patients were not as old and only a few were nursing home residents. However, long-term mortality remained poor in our cohort, as shown in other studies. ${ }^{14}$

Age is considered a major predictor of poor outcome in many illnesses, including pneumonia, and is an important component of the PPI risk classification system. In our study, almost half of the episodes designated Class 3 and Class 4 did not have any PPI-specific comorbidities. Rello et al. found that the presence of complications and the severity of illness at initial presentation were the major variables affecting outcome in older adults with severe CAP; with the exception of immunosuppression, comorbidities did not influence outcomes. ${ }^{13}$ Given the shorter length of stay and a very low mortality, Class 3 patients may have been an appropriate target for outpatient management.

We noted only two patients with S. pneumoniae pneumonia and a greater than expected occurrence of gramnegative bacilli. Bates et al. noted that gram-negative bacilli were the causative agents of pneumonia in 12 of 154 $(8 \%)$ patients, and gram-positive cocci accounted for 22 of $154(14 \%) .{ }^{17}$ These contrasts could be explained by the differences in patient population studied or methods of obtaining the etiologic agent. As in other studies, the overall yield of blood cultures remained low. ${ }^{12,13}$ However, our study was not designed to optimize the collection of sputum or to define the etiologic cause of pneumonia.

The practical utility of PPI remains to be determined. PPI requires diagnostic testing including renal function, electrolytes, glucose, hemoglobin, and arterial blood gas measurements. Although these tests were performed on admission for most of our patients evaluated in the emergency room, the decision for admission and the necessity for a monitored bed is usually made before some of the results are available: a situation that is even more applicable in nursing homes or outpatient clinics. In a recent study, it was demonstrated that variables usually available during initial evaluation (e.g., age, presence of a comorbid condition, altered motor response to verbal commands, an abnormal vital sign, and serum creatinine $>1.5 \mathrm{mg} / \mathrm{dl}$ ) were independently associated with mortality in older adults. ${ }^{18} \mathrm{~A}$ discriminant rule was developed using these variables. Other outcomes, such as length of stay, development of complications, transfer to an intensive care unit, and impairment in functional status, were not assessed. Although not within the scope of this study, it would be interesting to compare these two severity indices in a prospective fashion.

In conclusion, we found that the higher classes of PPI could accurately identity older adults at higher risk of developing complications and mortality and also predicted the individuals with a longer length of stay. However, further prospective studies are required to further define the bedside clinical findings most predictive of mortality in older adults prospectively; the relative significance of age, comorbidities, and severity at presentation; and the role of functional status on the clinical outcome from CAP.

\section{ACKNOWLEDGMENTS}

The authors wish to thank Dr. Carol Kauffman for her suggestions and critical review of this manuscript.

Table 3. In-Hospital, 30-Day, and Long-Term Mortality by Pneumonia Prognosis Index (PPI) Class

PPI Class (number of episodes) In-Hospital Mortality $n(\%) \quad$ Mortality at 30 Days $n(\%) \quad$ Mortality at Long-Term Follow-Up* $n(\%)$

Class 2 (3)

Class $3(20)$

Class 4 (46)

Class 5 (32)

Total (101)

\section{0}

$1(0.5)$

3 (6.5)

4 (12.5)

8 (8)
0

$1(0.5)$

5 (10.8)

8 (25)

14 (14)
0

$20(63)$

37 (37)

*Average duration of follow-up was 13 months. 


\section{REFERENCES}

1. Van Nostrand JF, Furner SE, Suzman R. Health data on older Americans: United States, 1992. Vital Health Stat 3 1993;(27):9-21.

2. Niederman MS, Bass JB, Campbell GD et al. Guidelines for the initial management of adults with community-acquired pneumonia: Diagnosis, assessment of severity, and initial antimicrobial therapy. Am Rev Respir Dis 1993; 148:1418-1426.

3. Graves EJ. National Hospital Discharge Survey: Annual summary, 1993. Vital Health Stat 13 1995;(121):1-63.

4. Kochanek KD, Maurer JD, Rosenberg HM. Causes of death contributing to changes in life expectancy: United States, 1984-89. Vital Health Stat 20 1994;(23):1-25.

5. Gillick MR, Serrell NA, Gillick LS. Adverse consequences of hospitalization in the elderly. Soc Sci Med 1982;16:1033-1038.

6. Hirsch $\mathrm{CH}$, Sommers L, Olsen A et al. The natural history of functional morbidity in hospitalized older patients. J Am Geriatr Soc 1990;38:1296-1303.

7. Inouye SK, Wagner DR, Acampora D et al. A predictive index for functional decline in hospitalized elderly medical patients. J Gen Intern Med 1993;8:645-652.

8. Fine MJ, Auble TE, Yealy DM et al. A prediction rule to identify low-risk patients with community acquired pneumonia. N Engl J Med 1997;336:243-250.

9. McGeer A, Campbell B, Emori TG et al. Definitions of infection for surveillance in long-term care facilities. Am J Infect Control 1991;19:1-7.

10. Mylotte JM, Naughton B, Saludades C et al. Validation and application of the pneumonia prognosis index to nursing home residents with pneumonia. J Am Geriatr Soc 1998;46:1538-1544.

11. Meehan TP, Fine MJ, Krumholz HM et al. Quality of care, process and outcomes in elderly patients with pneumonia. JAMA 1997;278:2080-2084.

12. Meehan TP, Chua-Reyes JM, Tate J et al. Process of care performance, patient characteristics, and outcomes in elderly patients hospitalized with communityacquired or nursing-home acquired pneumonia. Chest 2000;117:1378-1385.

13. Rello J, Rodriguez R, Jubert $P$ et al. Severe community-acquired pneumonia in the elderly-epidemiology and prognosis. Clin Infect Dis 1996;23:723-728.

14. Koivula I, Sten M, Makela PH. Prognosis after community acquired pneumonia in the elderly. A population-based 12 year follow-up study. Arch Intern Med 1999;159:1550-1555.

15. Hedlund JU, Ortqvist AB, Kalin ME et al. Factors of importance for the longterm prognosis after hospital-treated pneumonia. Thorax 1993;48:785-789.

16. Sikka P, Jaafar WM, Bozkanat E et al. A comparison of severity of illness scoring systems for elderly patients with severe pneumonia. Intensive Care Med 2000;26:1803-1810.

17. Bates JH, Campbell GD, Barron AL et al. Microbial etiology of acute pneumonia in hospitalized patients. Chest 1992;101:1005-1012.

18. Conte HA, Chen Y, Mehal W et al. A prognostic rule for elderly patients admitted with community-acquired pneumonia. Am J Med 1999;106:20-28. 